\title{
THE NEEDS AND REQUIREMENTS FROM \\ THE STANDPOINT OF THE ULTRAVIOLET \\ SOLAR OBSERVATIONS
}

\author{
E. M. REEVES \\ (Harvard College Observatory)
}

Extending from the present to the early part of 1969 there are three Orbiting Solar Observatories to be launched, and these will all be capable of constructing spectroheliograms of the Sun in solar emission lines of the EUV and X-ray region. The recently launched and highly successful OSO-III has obtained EUV and X-ray spectra with high-time resolution, but without spatial resolution on the solar disk. The later OSO satellites will provide spatial resolution of $1^{\prime}$ of arc to $30^{\prime \prime}$ of arc, and will provide the basis for the extension to even higher spatial resolution in the future.

The comparatively short periods covered by these satellites, coupled with a real probability of only partial success, make it particularly important to obtain the fullest possible use of the data by implementing a complementary and simultaneous series of ground-based observations.

The data from solar satellite experiments are frequently capable of providing information in several fields other than that for which they were initially designed. Thus the correlation of data from such satellites can be useful in the fuller interpretation of the experiments on the interplanetary medium, and the structure of the Earth's atmosphere, as well as for solar experimentation.

At the present time the Interplanetary Monitoring Probe (IMP-F) is successfully recording data on the hydrogen and helium ion concentrations in a highly eccentric orbit, which extends at certain times into the region of the undisturbed solar wind. The OSO-D satellite which we currently expect to be launched in October, will probably be in orbit at the same time with the capability of producing spectroheliograms in $\mathrm{HI}, \mathrm{HeI}$ and HeII lines, as well as other solar emission lines with 1' of arc spatial resolution. The experimenters on the two satellites have agreed to program the observations of the two satellites so that enhancements of $\mathrm{H}$ and $\mathrm{He}$ ions detected in the solar wind can be correlated with the spatial distribution of enhancements of optical radiation in the same elements in various sectors of the solar disk several days previously.

There seems to be a good probability that two OSO satellites, namely OSO-III and OSO-IV, will also be in orbit together in the next few months, and it will then be possible to compare the interesting whole Sun enhancements in the shorter wavelengths and X-ray regions which have been reported in Commission 44 with spatially

Perek (ed.), Highlights of Astronomy, 538-540. (c) I.A.U. 
resolved enhancements in the OSO-IV experiments. Simultaneous comparisons of absolute fluxes as well as time variations can be made. The instrument packages on each satellite generally cover a fairly wide range of wavelengths, and here again intercomparison of data among experimenters is highly desirable and profitable.

The observation of emissions of the EUV and X-ray fluxes from the Sun can be interpreted to yield information on the structure and variation of the Earth's atmosphere and hence may be correlated with effects in the terrestrial atmosphere. Both the present and the next OSO have the capability to observe the absorption of solar ultraviolet lines as a function of height in the Earth's atmosphere during sunrise and sunset portions of the orbit. These observations have been referred to by Hinteregger during the present meetings of Commission 44 and will be continued by our own experiment on OSO-IV.

The third area of coordination is that of simultaneous solar observations from the ground and from space, and is of most immediate interest to the present discussion. A general program of ground-based observations such as fairly continuous $\mathrm{HI}$ and Ca II spectroheliograms from stations around the world, and at least daily coronagraph observations with good spatial resolution to be used for both flare prediction and data correlation, will all be required for the optimum use of the satellite ultraviolet data as will communication with the daily solar activity and flare warning systems presently in operation. Coordination is now being planned for the OSO-IV satellite, and a number of observatories have already been contacted. There is a particular need to extend the coverage of the ground-based spectroheliograms to include more lines, and especially to increase the number of observing sites to extend the amount of coverage as well as to compensate for seasonal and weather variabilities at existing facilities.

The OSO-IV satellite will be able to construct spectroheliograms in lines of OI-VI, NII-V, Si III and XII, CII and III, HeI and II, NevIII, Mgx, SixII and the lines of the hydrogen Lyman series as well as the Lyman continuum.

A number of special programs are being arranged in order to obtain specific groundbased spectroheliograph observations simultaneously with OSO-IV observations. Spectroheliograms in lines of $\mathrm{C}, \mathrm{N}, \mathrm{O}, \mathrm{He}, \mathrm{H}$, etc., will be made on the ground at the same time that observations in various stages of ionization of the same element are being observed from the spacecraft. This is being done in order to evaluate the extent to which the observations from solar satellites indicate unique variations or structures and to assess the extent to which the ground-based observations can be used for those extended periods when satellite observations will not be available. We solicit the cooperation of any observatories in this venture and welcome any suggestions from other astronomers on how our satellite observations can be scheduled to support specific researches which will be in progress this autumn and winter.

A manned solar observatory, referred to as ATM (Apollo Telescope Mount), is being prepared at the present time for several launches in the period 1969-71. This 
manned satellite will use an astronaut observer to program the solar observations to take maximum advantage of the activity on the disk. Coronagraphs, UV spectrographs and spectrometers, photographic and photoelectric spectroheliograms and $\mathrm{X}$-ray and $\mathrm{H} \alpha$ telescopes are all included in the payload. Most of the experiments employ recoverable photographic records although several of the experiments, including our own, are photoelectric. This mission will be limited to $4-8$ weeks and the maximum possible cordination with ground-based observations will be required to make optimum use of these observations. Primarily required are extended temporal coverage by coronagraphs, magnetographs and spectroheliographs in the lines $I$ have already mentioned, and the establishment of an effective means of communications between the participating observatories and the satellite experimenters. The spatial resolution from the ATM experiments will be approximately $5^{\prime \prime}$ of arc, which begins to become comparable with the resolution which can be obtained from the ground at much longer wavelengths. The intercomparison can then be made at about the same resolution and will be much more meaningful.

The prediction of regions on the solar disk which are likely to exhibit larger flare activity as well as estimates of the onset time for such events will be necessary if the ATM experiments are to have any hope of catching a moderate to large flare, particularly in the important initial phases of the flare. Magnetograph observations with good accuracy and wider global coverage seem to offer some chance of observing such an event, if adequate coordination and communication can be established.

The relatively short lifetime of the ATM satellite experiments underlines the need for a careful pre-arrangement of the observing program of ground-based observatories to take maximum advantage of the data from the space experiments. 